\title{
Relationships between factors of canonical central series of Leibniz algebras
}

\author{
Leonid A. Kurdachenko ${ }^{1}$ • Javier Otal ${ }^{2}$. \\ Alexander A. Pypka ${ }^{1}$
}

Received: 24 July 2015 / Revised: 13 December 2015 / Accepted: 23 December 2015 /

Published online: 19 January 2016

(C) Springer International Publishing AG 2016

\begin{abstract}
The aim of this paper is to show a close relationship between the factoralgebras by the terms of the upper central series $\zeta_{n}(L)$ of a Leibniz algebra $L$ and the terms of its lower central series $\gamma_{n}(L)$. Specifically we show that finiteness of codimension of some $\zeta_{k}(L)$ implies finiteness of dimension of $\gamma_{k+1}(L)$ and give explicit bounds for this dimension. We also improve this in the case $k=1$, which corresponds to the center and commutator subalgebra of the algebra, respectively. These results are analogous to the results that have been obtained for groups and Lie algebras.
\end{abstract}

Keywords Leibniz algebra $\cdot$ Lie algebra $\cdot$ Center $\cdot$ Commutator subalgebra $\cdot$ Lower and upper central series of a Leibniz algebra

Mathematics Subject Classification $17 \mathrm{~A} 32 \cdot 17 \mathrm{~A} 60 \cdot 17 \mathrm{~B} 30$

\footnotetext{
Javier Otal was supported by the Department of I+D of the Government of Aragón (Spain) and FEDER funds from the European Union.

Javier Otal

otal@unizar.es

Leonid A. Kurdachenko

lkurdachenko@gmail.com

Alexander A. Pypka

pypka@ua.fm

1 Faculty of Mechanics and Mathematics, National University of Dnepropetrovsk, 18 Kazakova St., 49000 Dnepropetrovsk, Ukraine

2 Department of Mathematics-IUMA, University of Zaragoza, Pedro Cerbuna 12, 50009 Zaragoza, Spain
} 


\section{Introduction}

An algebra $L$ over a field $F$ is said to be a (left) Leibniz algebra if it is endowed with a multiplication (or bracket) $[\cdot, \cdot]$ that satisfies the so-called Leibniz identity

$$
[[a, b], c]=[a,[b, c]]-[b,[a, c]], \quad a, b, c \in L .
$$

A right Leibniz algebra is defined similarly. Leibniz algebras arise as a generalization of Lie algebras. Indeed, if $L$ is a Leibniz algebra such that $[a, a]=0$ for every element $a \in L$, then it is not hard to check that $L$ is a Lie algebra. Therefore we may think of Leibniz algebras as a non-anticommutative analog of Lie algebras.

Leibniz algebras were first introduced and investigated in the papers of A.M. Blokh [8-10], where he called them D-algebras. However, they did not receive a worthy attention immediately. Later these algebras were independently rediscovered by J.L. Loday [17], who called them Leibniz algebras, since it was Leibniz who proved the Leibniz rule for differentiation of functions. Leibniz algebras appeared to be related in a natural way with several topics such as differential geometry, homological algebra, classic algebraic topology, algebraic $K$-theory, loop spaces, noncommutative geometry, etc. They also found some applications in physics (see $[11,13,14]$, for example). Other papers concerning Leibniz algebras are devoted to the study of homological problems (see [12,16,18,21], for example).

Structural properties of Leibniz algebras are well studied. In most cases analogs of important results from the theory of Lie algebras for them are straightforward, but some of them have a specificity. This is the case of nilpotent Leibniz algebras. The concept of nilpotency plays a very important role for Lie algebras, associative algebras, groups and other algebraic structures. The study of nilpotent Leibniz algebras started in the paper of Sh.A. Ayupov and B.A. Omirov [3] and continued in the papers $[1,2,4,7,15,20]$, sometimes under different approaches. The concept of nilpotency of Leibniz algebras has a serious difference from the nilpotency of Lie algebras. In all papers mentioned above it relies on the concept of lower central series. To define it as well as other concepts we recall some standard definitions and notation.

Let $L$ be a Leibniz algebra over a field $F$. If $A$ and $B$ are subspaces of $L$, then $[A, B]$ is the subspace generated by $\{[a, b]: a \in A, b \in B\}$. A subspace $A$ of $L$ is called a subalgebra of $L$ if $[A, A] \leqslant A$. A subalgebra $A$ is called a left ideal of $L$ (respectively, a right ideal of $L$ ) if $[L, A] \leqslant A$ (respectively, $[A, L] \leqslant A$ ), and it is called an (two-sided) ideal of $L$ if it is both a left ideal and a right ideal. If $A$ is an ideal of $L$, then the factor subspace $L / A$ is in fact a Leibniz algebra. We denote by $\operatorname{Leib}(L)$ is the subspace generated by $\{[a, a]: a \in L\}$. Leib $(L)$ is an ideal of $L$ (called the Leibniz kernel of $L$ ); if $H$ is an ideal of $L$ such that $L / H$ is a Lie algebra, then Leib $(L) \leqslant H$. Note the following important property:

$$
[[a, a], x]=0 \quad \text { for arbitrary elements } a, x \in L \text {. }
$$

If $A$ and $B$ are ideals of a Leibniz algebra $L$, then $[A, B]$ need not be an ideal of $L$; a specific example can be found in [6]. However, if $H$ is an ideal, then $[H, H]$ is an ideal, which is important in what follows. 
The lower central series of $L$

$$
L=\gamma_{1}(L) \geqslant \gamma_{2}(L) \geqslant \cdots \geqslant \gamma_{\alpha}(L) \geqslant \gamma_{\alpha+1}(L) \geqslant \cdots \geqslant \gamma_{\delta}(L)
$$

is defined inductively by the rules: $\gamma_{1}(L)=L, \gamma_{\alpha+1}(L)=\left[L, \gamma_{\alpha}(L)\right]$ for every ordinal $\alpha$ and $\gamma_{\lambda}(L)=\bigcap_{\mu<\lambda} \gamma_{\mu}(L)$ for every limit ordinal $\lambda$. It is rather easy to show that every term of this series is an ideal of $L$. The last term $\gamma_{\delta}(L)$ of the series is called the lower hypercenter of $L$. We note that $\gamma_{\delta}(L)=\left[L, \gamma_{\delta}(L)\right]$. Also if $\alpha=k$ is a positive integer, then $\gamma_{k}(L)=[L,[L,[L, \ldots] \ldots]]$ is a left normed product of $k$ copies of $L$.

The Leibniz algebra $L$ is called nilpotent if there exists a positive integer $k$ such that $\gamma_{k}(L)=\{0\}$. More precisely, $L$ is said to be nilpotent of nilpotency class $c$ if $\gamma_{c+1}(L)=\{0\}$ but $\gamma_{c}(L) \neq\{0\}$. We denote the nilpotency class of $L$ by $\operatorname{ncl}(L)$. The concept of lower central series is specific not only for Leibniz algebras but also for other algebraic structures such as Lie algebras and groups. However, for the latter structures there exists another canonical series, which is dual to the lower central series, the upper central series. The case of Leibniz algebras has essential differences that are worth to study, for example, the notion of the center.

By definition, the left center $\zeta^{1}(L)$ and the right center $\zeta^{\mathrm{r}}(L)$ of $L$ are

$$
\begin{aligned}
& \zeta^{1}(L)=\{x \in L:[x, y]=0 \text { for each } y \in L\}, \\
& \zeta^{\mathrm{r}}(L)=\{x \in L:[y, x]=0 \text { for each } y \in L\} .
\end{aligned}
$$

Clearly, $\zeta^{1}(L)$ is an ideal and $\operatorname{Leib}(L) \leqslant \zeta^{1}(L)$ and so $L / \zeta^{1}(L)$ is a Lie algebra. On the other hand, the right center is a subalgebra of $L$ but it need not be an ideal. In general, the left and right centers are different. In Sect. 2, we will present the corresponding example. The center $\zeta(L)$ of $L$ is the intersection of the left and right centers, that is

$$
\zeta(L)=\{x \in L:[x, y]=0=[y, x] \text { for every } y \in L\} .
$$

The center $\zeta(L)$ is an ideal of $L$, and thus the factor-space $L / \zeta(L)$ is an algebra.

The upper central series of $L$,

$$
\{0\}=\zeta_{0}(L) \leqslant \zeta_{1}(L) \leqslant \cdots \leqslant \zeta_{\alpha}(L) \leqslant \zeta_{\alpha+1}(L) \leqslant \cdots \leqslant \zeta_{\eta}(L)=\zeta_{\infty}(L),
$$

is defined inductively by the rules $\zeta_{1}(L)=\zeta(L), \zeta_{\alpha+1}(L) / \zeta_{\alpha}(L)=\zeta\left(L / \zeta_{\alpha}(L)\right)$ for every ordinal $\alpha$ and $\zeta_{\lambda}(L)=\bigcup_{\mu<\lambda} \zeta_{\mu}(L)$ for every limit ordinal $\lambda$. Note that, by construction, every term of this series is an ideal of $L$. The last term $\zeta_{\infty}(L)$ of the series is called the upper hypercenter of $L$. We $\operatorname{set} \operatorname{zl}(L)$ to denote the length of this series.

For Lie algebras $L$, the fact that $\gamma_{c+1}(L)=\{0\}$ is equivalent to $\zeta_{c}(L)=L$, that is for a nilpotent Lie algebra the lower and upper central series have the same length (similar facts hold for groups). In this paper we will show that this remains true for Leibniz algebras. The case of finite upper or lower central series naturally raises the question of investigation of relationships between $L / \zeta_{k}(L)$ and $\gamma_{k+1}(L)$. If $L$ is a 
Lie algebra such that $L / \zeta_{k}(L)$ is finitely dimensional, I.N. Stewart [22, Theorem 5.2] showed that $\gamma_{k+1}(L)$ is also finitely dimensional. For groups, the corresponding result was obtained earlier by R. Baer [5]. Our first main result is an analog of these theorems.

Theorem A Let L be a Leibniz algebra over a field $F$. If $\operatorname{codim}_{F} \zeta_{k}(L)=d$ is finite for some $k \geqslant 1$, then $\operatorname{dim}_{F} \gamma_{k+1}(L) \leqslant 2^{k-1} d^{k+1}$.

As a corollary we may improve the bound for the dimension of $\gamma_{k+1}(L)$ for Lie algebras, thus completing the result of Stewart mentioned above.

Corollary A1 Let L be a Lie algebra over a field $F$. If $\operatorname{codim}_{F} \zeta_{k}(L)=d$ is finite for some $k \geqslant 1$, then $\operatorname{dim}_{F} \gamma_{k+1}(L) \leqslant d^{k-1}(d-1) / 2$.

An important specific case in these results is the case $k=1$ when the center of the Leibniz algebra $L$ has finite codimension. For Lie algebras the following result is well-known (see [23], for example): if $L$ is a Lie algebra over a field $F$ and the factor-algebra $L / \zeta(L)$ has finite dimension $d$, then $\operatorname{dim}_{F}[L, L] \leqslant d(d+1) / 2$.

For groups the corresponding result was proved much earlier and makes up one of the most outstanding results of the theory: if $C$ is a central subgroup of a group $G$ such that $G / C$ is finite, then the derived subgroup $[G, G]$ is finite. In this formulation, it appears for the first time in the paper of B.H. Neumann [19] but the result was also obtained by R. Baer [5]. For Leibniz algebras we obtain the following analogous results.

Theorem B Let L be a Leibniz algebra over a field $F$. If $\operatorname{codim}_{F} \zeta^{1}(L)=d$ and $\operatorname{codim}_{F} \zeta^{\mathrm{r}}(L)=r$ are finite, then $\operatorname{dim}_{F}[L, L] \leqslant d(d+r)$.

In this setting we can raise the question of studying the above result when only one of the above codimensions is finite. In Sect. 3, we construct an example that shows that the answer to the question "If $\operatorname{codim}_{F} \zeta^{1}(L)$ is finite, is $\operatorname{dim}_{F}[L, L]$ finite?" is negative. Despite this, other cases are more favourable.

Corollary B1 Let L be a Leibniz algebra over a field $F$. If $\operatorname{codim}_{F} \zeta(L)=d$ is finite, then $\operatorname{dim}_{F}[L, L] \leqslant d^{2}$.

Corollary B2 Let L be a Leibniz algebra over a field $F$. If $\operatorname{codim}_{F} \zeta(L)=d$ is finite, then $\operatorname{dim}_{F} \operatorname{Leib}(L) \leqslant d(d-1) / 2$.

\section{Auxiliary results}

First we construct an example of a Leibniz algebra in which left and right centers are different. Actually they will have different dimensions.

Example 2.1 Let $F$ be a field. Consider a vector space $L$ over $F$ of dimension 4 , say $L=F e_{1} \oplus F e_{2} \oplus F e_{3} \oplus F e_{4}$, and define an operation $[\cdot, \cdot]$ as follows:

$$
\begin{aligned}
& {\left[e_{1}, e_{1}\right]=e_{2}, \quad\left[e_{1}, e_{2}\right]=-e_{2}-e_{3}, \quad\left[e_{1}, e_{3}\right]=e_{2}+e_{3},} \\
& {\left[e_{1}, e_{4}\right]=0, \quad\left[e_{2}, e_{1}\right]=\left[e_{3}, e_{1}\right]=0, \quad\left[e_{4}, e_{1}\right]=e_{2}+e_{3} \text {, }} \\
& {\left[e_{j}, e_{k}\right]=0 \quad \text { for all } j, k \in\{2,3,4\} \text {. }}
\end{aligned}
$$


It is not hard to check that this operation defines a Leibniz algebra. We can also see that $\zeta^{\mathrm{r}}(L)=F e_{4}$ and so $\zeta^{\mathrm{r}}(L)$ is not an ideal. Furthermore, $\zeta^{\mathrm{l}}(L)=F e_{2} \oplus F e_{3}$, and then $\zeta^{\mathrm{r}}(L) \cap \zeta^{\mathrm{l}}(L)=\{0\}, \operatorname{dim}_{F} \zeta^{\mathrm{r}}(L)=1$ and $\operatorname{dim}_{F} \zeta^{1}(L)=2$. We also note that $[L, L]=\operatorname{Leib}(L)=\zeta^{1}(L)$.

Now we collect some technical results needed in the sequel.

Proposition 2.2 Let $L$ be a Leibniz algebra over a field $F$ and $H$ an ideal of $L$. Then

(i) $[H, H]$ is an ideal of $L$;

(ii) $[L, H]$ is a subalgebra of $L$;

(iii) $[H, L]$ is a subalgebra of $L$;

(iv) $[L, H]+[H, L]$ is an ideal of $L$;

(v) $\left[\gamma_{j}(H), \gamma_{k}(H)\right] \leqslant \gamma_{j+k}(H)$ for all positive integers $j, k$;

(vi) $\gamma_{j}(H)$ is an ideal of $L$ for every positive integer $j$, in particular, $\gamma_{j}(L)$ is an ideal of $L$ for every positive integer $j$; and

(vii) $\gamma_{j}\left(\gamma_{k}(H)\right) \leqslant \gamma_{j k}(H)$ for all positive integers $j, k$.

Proof The proof of (i)-(iv) is not complicated and we omit it.

(v) We proceed by induction on $j$. If $j=1$, the result follows from the definition. Suppose now that $j>1$ and we have already proved the inclusion

$$
\left[\gamma_{m}(H), \gamma_{k}(H)\right] \leqslant \gamma_{m+k}(H) \quad \text { for all } m<j \text {. }
$$

We have $\gamma_{j}(H)=\left[H, \gamma_{j-1}(H)\right]$. Pick $x \in H, y \in \gamma_{j-1}(H)$ and $z \in \gamma_{k}(H)$. We have

$$
[[x, y], z]=[x,[y, z]]-[y,[x, z]]
$$

Since $[y, z] \in\left[\gamma_{j-1}(H), \gamma_{k}(H)\right]$, by induction, $[y, z] \in \gamma_{j-1+k}(H)$ whence $[x,[y, z]] \in\left[H, \gamma_{j-1+k}(H)\right]=\gamma_{j+k}(H)$. Further

$$
[y,[x, z]] \in\left[\gamma_{j-1}(H),\left[H, \gamma_{k}(H)\right]\right]=\left[\gamma_{j-1}(H), \gamma_{k+1}(H)\right] .
$$

Applying induction,

$$
\left[\gamma_{j-1}(H), \gamma_{k+1}(H)\right] \leqslant \gamma_{j-1+k+1}(H)=\gamma_{j+k}(H)
$$

Hence $[y,[x, z]] \in \gamma_{j+k}(H)$, which gives $\left[\gamma_{j}(H), \gamma_{k}(H)\right] \leqslant \gamma_{j+k}(H)$.

(vi) Again we proceed by induction on $j$. If $j=1$, the result follows from definitions. Suppose now that $j>1$ and we have already proved that $\gamma_{m}(H)$ is an ideal of $L$ for all $m<j$. We have $\gamma_{j}(H)=\left[H, \gamma_{j-1}(H)\right]$. Pick $x \in H, y \in \gamma_{j-1}(H)$ and $z \in L$. We have $[[x, y], z]=[x,[y, z]]-[y,[x, z]]$. By induction, $[y, z] \in \gamma_{j-1}(H)$ and so $[x,[y, z]] \in\left[H, \gamma_{j-1}(H)\right]=\gamma_{j}(H)$. Since $H$ is an ideal of $L,[x, z] \in H$ and then $[y,[x, z]] \in\left[\gamma_{j-1}(H), H\right]$. By induction,

$$
\left[\gamma_{j-1}(H), H\right]=\left[\gamma_{j-1}(H), \gamma_{1}(H)\right]=\gamma_{j}(H)
$$


Hence $[[x, y], z] \in \gamma_{j}(H)$. Similarly, $[x,[z, y]] \in \gamma_{j}(H)$.

(vii) One more time we proceed by induction on $j$. Since $\gamma_{1}\left(\gamma_{k}(H)\right)=\gamma_{k}(H)$, the case $j=1$ is trivial. Suppose now that $j>1$ and we have already proved the inclusion $\gamma_{m}\left(\gamma_{k}(H)\right) \leqslant \gamma_{m k}(H)$ for all $m<j$. We have

$$
\gamma_{j}\left(\gamma_{k}(H)\right)=\left[\gamma_{j-1}\left(\gamma_{k}(H)\right), \gamma_{k}(H)\right] \leqslant\left[\gamma_{j k-k}(H), \gamma_{k}(H)\right]
$$

Applying (iii), we obtain the inclusion

$$
\gamma_{j}\left(\gamma_{k}(H)\right) \leqslant \gamma_{j k-k+k}(H)=\gamma_{j k}(H)
$$

and we are done.

An ascending series of ideals of a Leibniz algebra $L$

$$
\{0\}=C_{0} \leqslant C_{1} \leqslant \cdots \leqslant C_{\alpha} \leqslant C_{\alpha+1} \leqslant \cdots \leqslant C_{\rho}=L
$$

is said to be central if $C_{\alpha+1} / C_{\alpha} \leqslant \zeta\left(L / C_{\alpha}\right)$ for every ordinal $\alpha<\rho$. In other words, $\left[C_{\alpha+1}, L\right],\left[L, C_{\alpha+1}\right] \leqslant C_{\alpha}$ for every ordinal $\alpha<\rho$.

As in other structures, for example in groups, central series of finite length characterizes nilpotency as the following result shows.

Proposition 2.3 Let L be a Leibniz algebra over a field $F$ and

$$
\{0\}=C_{0} \leqslant C_{1} \leqslant \cdots \leqslant C_{n}=L
$$

be a central series of L of finite length. Then we have

(i) $\gamma_{j}(L) \leqslant C_{n-j+1}$ for every $1 \leqslant j \leqslant n+1$. In particular, $\gamma_{n+1}(L)=\{0\}$; and

(ii) $C_{j} \leqslant \zeta_{j}(L)$ for every $0 \leqslant j \leqslant n$. In particular, $\zeta_{n}(L)=L$.

Proof (i) We proceed by induction on $j$. If $j=2$, we have

$$
\gamma_{2}(L)=[L, L]=\left[L, C_{n}\right] \leqslant C_{n-1} .
$$

Suppose now that $j>2$ and we have already proved the inclusion $\gamma_{m}(L) \leqslant C_{n-m+1}$ for all $m<j$. Then $\gamma_{j}(L)=\left[L, \gamma_{j-1}(L)\right] \leqslant\left[L, C_{n-j+1+1}\right] \leqslant C_{n-j+1}$ and we are done.

(ii) We proceed again by induction on $j$. If $j=1$, we have $\left[C_{1}, L\right]=\left[L, C_{1}\right]=\{0\}$, which shows that $C_{1} \leqslant \zeta_{1}(L)$. Suppose now that $j>1$ and we have already proved the inclusion $C_{m} \leqslant \zeta_{m}(L)$ for all $m<j$. It follows that

$$
\left[C_{j}, L\right],\left[L, C_{j}\right] \leqslant C_{j-1} \leqslant \zeta_{j-1}(L) .
$$

Then

$$
\left[C_{j}+\zeta_{j-1}(L), L\right],\left[L, C_{j}+\zeta_{j-1}(L)\right] \leqslant \zeta_{j-1}(L)
$$


which gives the inclusions

$$
\left(C_{j}+\zeta_{j-1}(L)\right) / \zeta_{j-1}(L) \leqslant \zeta L / \zeta_{j-1}(L)=\zeta_{j}(L) / \zeta_{j-1}(L)
$$

and we are done.

This result has the following consequences.

Corollary 2.4 Let $L$ be a Leibniz algebra over a field $F$ and suppose that L has a finite central series

$$
\{0\}=C_{0} \leqslant C_{1} \leqslant \cdots \leqslant C_{n}=L .
$$

Then $L$ is nilpotent and $\operatorname{ncl}(L) \leqslant n$. Furthermore, the upper central series of $L$ has finite length $\mathrm{zl}(L) \leqslant n$. Moreover, $\operatorname{ncl}(L)=\mathrm{zl}(L)$.

The above corollary shows that the Leibniz algebra $L$ is nilpotent if and only if there is a positive integer $k$ such that $L=\zeta_{k}(L)$. The least positive integer with this property coincides with the nilpotency class of $L$.

Corollary 2.5 Let L be a Leibniz algebra over a field $F$ and $H$ an ideal of $L$. If the factor-algebra $L / H$ is nilpotent of nilpotency class at most $n$, then $\gamma_{n+1}(L) \leqslant H$.

With some extra work we may prove something more.

Corollary 2.6 Let $L$ be a Leibniz algebra over a field $F$ and $H$ an ideal of L. Then $\gamma_{n}(L / H)=\left(\gamma_{n}(L)+H\right) / H$ for every positive integer $n$.

Proof We proceed by induction on $n$. If $n=1$, we have $\gamma_{1}(L / H)=L / H=$ $\gamma_{1}(L) / H$. Suppose now that $n>1$ and we have already proved $\gamma_{m}(L / H)=$ $\left(\gamma_{m}(L)+H\right) / H$ for all $m<n$. Then

$$
\begin{aligned}
\gamma_{n}(L / H) & =\left[L / H, \gamma_{n-1}(L / H)\right]=\left[L / H,\left(\gamma_{n-1}(L)+H\right) / H\right] \\
& =\left(\left[L, \gamma_{n-1}(L)\right]+H\right) / H=\left(\gamma_{n}(L)+H\right) / H
\end{aligned}
$$

as required.

\section{Proof of Theorem B}

We start by describing an example mentioned in Introduction.

Example 3.1 Let $F$ be a field. We consider an $F$-vector space with the form

$$
L=F e_{1} \oplus F e_{2} \oplus Z
$$

where the subspace $Z$ has a countable basis, say $\left\{z_{n}: n \geqslant 1\right\}$. We define $\left[z_{n}, x\right]=0$ for every $x \in L$ and

$$
\left[e_{1}, e_{1}\right]=\left[e_{2}, e_{2}\right]=\left[e_{1}, e_{2}\right]=\left[e_{2}, e_{1}\right]=z_{1}, \quad\left[e_{1}, z_{1}\right]=\left[e_{2}, z_{1}\right]=0 .
$$


Then we have

$0=\left[\left[e_{j}, e_{k}\right], e_{m}\right], \quad\left[e_{j},\left[e_{k}, e_{m}\right]\right]-\left[e_{k},\left[e_{j}, e_{m}\right]\right]=0-0=0, \quad j, k, m \in\{1,2\}$.

Taking into account the equalities

$$
\begin{aligned}
& 0=\left[\left[e_{1}, e_{2}\right], z\right]=\left[e_{1},\left[e_{2}, z\right]\right]-\left[e_{2},\left[e_{1}, z\right]\right], \\
& 0=\left[\left[e_{2}, e_{1}\right], z\right]=\left[e_{2},\left[e_{1}, z\right]\right]-\left[e_{1},\left[e_{2}, z\right]\right],
\end{aligned}
$$

we obtain $\left[e_{2},\left[e_{1}, z\right]\right]-\left[e_{1},\left[e_{2}, z\right]\right]=0$ for every $z \in Z$. Now we put

$$
\left[e_{1}, z_{j}\right]=z_{j}, \quad\left[e_{2}, z_{j}\right]=z_{j+1}, \quad j>1 .
$$

From these definitions we deduce

$$
\begin{array}{ll}
0=\left[\left[e_{j}, z\right], e_{k}\right], & {\left[e_{j},\left[z, e_{k}\right]\right]-\left[z,\left[e_{j}, e_{k}\right]\right]=\left[e_{j}, 0\right]-0=0,} \\
0=\left[\left[z, e_{j}\right], e_{k}\right], & {\left[z,\left[e_{j}, e_{k}\right]\right]-\left[e_{j},\left[z, e_{k}\right]\right]=0-\left[e_{j}, 0\right]=0}
\end{array}
$$

for all $j, k \in\{1,2\}$ and $z \in Z$. As we have seen above,

$$
\left[\left[e_{j}, e_{k}\right], z\right]=\left[e_{j},\left[e_{k}, z\right]\right]-\left[e_{k},\left[e_{j}, z\right]\right]
$$

for all $j, k \in\{1,2\}$ and $z \in Z$. Hence $L$ is a Leibniz algebra. By construction, $Z$ is the left center of $L$, the right center coincides with the center and this is equal to $F z_{1}$. It follows that the left center has finite codimension (and then infinite dimension) and the right center and the center have finite dimension.

Also, by construction, $[L, L]=Z$. Furthermore we have

$$
\begin{aligned}
& {\left[e_{1}+z_{1}, e_{1}+z_{1}\right]=\left[e_{1}, e_{1}\right]+\left[z_{1}, e_{1}\right]+\left[e_{1}, z_{1}\right]+\left[z_{1}, z_{1}\right]=z_{1},} \\
& {\left[e_{1}+z_{j}, e_{1}+z_{j}\right]=\left[e_{1}, e_{1}\right]+\left[z_{j}, e_{1}\right]+\left[e_{1}, z_{j}\right]+\left[z_{j}, z_{j}\right]=z_{1}+z_{j}}
\end{aligned}
$$

for all $j>1$. It follows that $\operatorname{Leib}(L)=Z$.

Example 3.1 shows that we may have a Leibniz algebra $L$ whose left center has finite codimension and whose derived subalgebra has infinite dimension. However, as we shall see, when the center of $L$ itself has finite codimension, the conclusion is rather different.

For the proof of our further results the following notion is fundamental. Let $L$ be a Leibniz algebra over a field $F, M$ a non-empty subset of $L$ and $H$ a subalgebra of $L$. We put

$$
\operatorname{Ann}_{H}^{1}(M)=\{a \in H:[a, M]=\{0\}\}, \quad \operatorname{Ann}_{H}^{\mathrm{r}}(M)=\{a \in H:[M, a]=\{0\}\} .
$$

These subsets are called the left and right annihilators of $M$ in the subalgebra $H$, respectively, and the intersection

$$
\operatorname{Ann}_{H}(M)=\operatorname{Ann}_{H}^{1}(M) \cap \operatorname{Ann}_{H}^{\mathrm{r}}(M)=\{a \in H:[a, M]=\{0\}=[M, a]\}
$$


is called the annihilator or centralizer of $M$ in the subalgebra $H$. It is not hard to see that all these subsets are subalgebras of $L$. Moreover, if $M$ is a left ideal of $L$ then $\operatorname{Ann}_{L}^{1}(M)$ is an ideal of $L$, and if $M$ is an ideal then $\operatorname{Ann}_{L}(M)$ is also an ideal of $L$. For example, the center of $L$ is the intersection of the annihilators of all elements of $L$.

Proposition 3.2 Let $L$ be a Leibniz algebra over a field $F$ and $H$ an ideal of $L$. Then the factor-algebra $L / \operatorname{Ann}_{L}^{1}(H)$ is isomorphic to a subalgebra of the algebra of derivations of $H$.

Proof If $a \in L$ we define a mapping $\iota_{a}: H \rightarrow H$ by the rule $\iota_{a}(x)=[a, x], x \in H$. It is rather easy to show that $\iota_{a}$ is a derivation of $H$ that satisfies $\beta \iota_{a}=\iota_{\beta a}, \iota_{a}+\iota_{b}=\iota_{a+b}$ and $\left[\iota_{a}, \iota_{b}\right]=\iota_{[a, b]}$, where $\beta \in F, a, b \in L$.

We consider now the mapping $\delta: L \rightarrow \operatorname{Der}(H)$ defined by the rule $\delta(a)=\iota_{a}$, $a \in L$. Applying the expressions proved in the above paragraphs, we have

$$
\begin{gathered}
\delta(\beta a)=\iota_{\beta a}=\beta \iota_{a}=\beta \delta(a), \quad \delta(a+b)=\iota_{a+b}=\iota_{a}+\iota_{b}=\delta(a)+\delta(b), \\
\delta([a, b])=\iota_{[a, b]}=\left[\iota_{a}, \iota_{b}\right]=[\delta(a), \delta(b)] .
\end{gathered}
$$

These equations show that the mapping $\delta$ is a homomorphism from the Leibniz algebra $L$ to the Lie algebra $\operatorname{Der}(H)$. Then $\operatorname{Im}(\delta)$ is a subalgebra of $\operatorname{Der}(H)$. Since $\operatorname{Im}(\delta) \cong$ $L / \operatorname{Ker}(\delta)$ and $\operatorname{Ker}(\delta)=\operatorname{Ann}_{L}^{1}(H)$, the result follows.

Proof of Theorem $B$ We have $L=\zeta^{1}(L) \oplus E$ for some subspace $E$. Choose in $E$ a basis, say $\left\{e_{1}, \ldots, e_{d}\right\}$. If $x, y \in L$, then we may write

$$
x=\alpha_{1} e_{1}+\cdots+\alpha_{d} e_{d}+z_{1}, \quad y=\beta_{1} e_{1}+\cdots+\beta_{d} e_{d}+z_{2}
$$

for suitable $\alpha_{1}, \ldots, \alpha_{d}, \beta_{1}, \ldots, \beta_{d} \in F$ and $z_{1}, z_{2} \in \zeta^{1}(L)$. Then we have

$$
[x, y]=\left[\sum_{j=1}^{d} \alpha_{j} e_{j}+z_{1}, \sum_{m=1}^{d} \beta_{m} e_{m}+z_{2}\right]=\sum_{1 \leqslant j, m \leqslant d} \alpha_{j} \beta_{m}\left[e_{j}, e_{m}\right]+\sum_{j=1}^{d} \alpha_{j}\left[e_{j}, z_{2}\right] .
$$

It follows that the subspace $S$ generated by the elements $\left[e_{j}, e_{m}\right], 1 \leqslant j, m \leqslant d$, and the subspaces $\left[e_{j}, \zeta^{1}(L)\right], 1 \leqslant j \leqslant d$, include $[L, L]$.

Put $Z=\zeta^{1}(L)$ and let $a$ be an arbitrary element of $L$. We define a mapping $\iota_{a}: Z \rightarrow Z$ by the rule $\iota_{a}(z)=[a, z], z \in Z$. By Proposition 3.2, this mapping is linear, $\operatorname{Im}\left(\iota_{a}\right)=[a, Z]$ and $\operatorname{Ker}\left(\iota_{a}\right)=\operatorname{Ann}_{Z}^{\mathrm{r}}(a)$. Hence

$$
[a, Z]=\operatorname{Im}\left(\iota_{a}\right) \cong_{F} Z / \operatorname{Ker}\left(\iota_{a}\right)=Z / \operatorname{Ann}_{Z}^{\mathrm{r}}(a) .
$$

Since $\zeta^{\mathrm{r}}(L) \leqslant \operatorname{Ann}_{L}^{\mathrm{r}}(a)$, we have $\operatorname{codim}_{F} \operatorname{Ann}_{L}^{\mathrm{r}}(a) \leqslant r$ and so

$$
\operatorname{dim}_{F}[a, Z]=\operatorname{dim}_{F} Z / \operatorname{Ann}_{Z}^{\mathrm{r}}(a) \leqslant r .
$$

In particular, $\operatorname{dim}_{F}\left[e_{j}, Z\right] \leqslant r$ for every $1 \leqslant j \leqslant d$. It follows that the subspace $S$ has dimension at most $d^{2}+d r=d(d+r)$, as required. 
Proof of Corollary B1 We have $\zeta(L)=\zeta^{1}(L) \cap \zeta^{\mathrm{r}}(L)$. Since $\operatorname{codim}_{F} \zeta^{1}(L)$ and $\operatorname{codim}_{F} \zeta^{\mathrm{r}}(L)$ are finite, $\operatorname{codim}_{F} \zeta(L)$ is finite. Then it suffices to take the proof of Theorem B into account to obtain Corollary B1.

Proof of Corollary B2 Put $K=\operatorname{Leib}(L)$ and $Z=\zeta(L)$. Clearly

$$
[L / K, L / K]=[L, L] / K, \quad Z / K \leqslant \zeta(L / K),
$$

therefore $\operatorname{codim}_{F} \zeta(L / K) \leqslant d$. The factor-algebra $L / K$ is in fact a Lie algebra, and $\operatorname{dim}_{F}[L / K, L / K] \leqslant d(d+1) / 2$. Hence

$$
\operatorname{dim}_{F} K=\operatorname{dim}_{F}[L, L]-\operatorname{dim}_{F}[L / K, L / K] \leqslant d^{2}-\frac{1}{2} d(d+1)=\frac{1}{2} d(d-1),
$$

as required.

\section{Proof of Theorem A}

To carry out the proof we need some auxiliary results.

Lemma 4.1 Let L be a Leibniz algebra over a field F. For every pair $k \geqslant j$ of positive integers we have $\left[\gamma_{j}(L), \zeta_{k}(L)\right],\left[\zeta_{k}(L), \gamma_{j}(L)\right] \leqslant \zeta_{k-j}(L)$.

Proof We proceed by induction on $j$. If $j=1$, we have $\left[\gamma_{1}(L), \zeta_{k}(L)\right]=$ $\left[L, \zeta_{k}(L)\right] \leqslant \zeta_{k-1}(L)$ and $\left[\zeta_{k}(L), \gamma_{1}(L)\right]=\left[\zeta_{k}(L), L\right] \leqslant \zeta_{k-1}(L)$. Suppose that $j>1$ and we have already proved that $\left[\gamma_{m}(L), \zeta_{k}(L)\right],\left[\zeta_{k}(L), \gamma_{m}(L)\right] \leqslant \zeta_{k-m}(L)$ for all $m<j$. We have $\gamma_{j}(L)=\left[L, \gamma_{j-1}(L)\right]$. Pick $x \in L, y \in \gamma_{j-1}(L)$ and $z \in \zeta_{k}(L)$. We have $[[x, y], z]=[x,[y, z]]-[y,[x, z]]$. Since $[y, z] \in\left[\gamma_{j-1}(L), \zeta_{k}(L)\right]$, by induction, $[y, z] \in \zeta_{k-j+1}(L)$, and so $[x,[y, z]] \in\left[L, \zeta_{k-j+1}(L)\right] \leqslant \zeta_{k-j}(L)$. Further,

$$
[y,[x, z]] \in\left[\gamma_{j-1}(L),\left[L, \gamma_{k}(L)\right]\right] \leqslant\left[\gamma_{j-1}(L), \zeta_{k-1}(L)\right]
$$

Since $k-1>j-1$, by induction, we obtain

$$
\left[\gamma_{j-1}(L), \zeta_{k-1}(L)\right] \leqslant \zeta_{k-1-j+1}(L)=\zeta_{k-j}(L)
$$

We have $[z,[x, y]]=[[z, x], y]+[x,[z, y]]$. Since $[z, x] \in \zeta_{k-1}(L)$,

$$
[[z, x], y] \in\left[\zeta_{k-1}(L), \gamma_{j-1}(L)\right]
$$

and so, by induction, $[[z, x], y] \in \zeta_{k-1-j+1}(L)=\zeta_{k-j}(L)$. Again, by induction, $[z, y] \in\left[\zeta_{k}(L), \gamma_{j-1}(L)\right] \leqslant \zeta_{k-j+1}(L)$. Then $[x,[z, y]] \in\left[L, \zeta_{k-j+1}(L)\right] \leqslant$ $\zeta_{k-j}(L)$ and we are done.

Lemma 4.2 Let $L$ be a Leibniz algebra over a field $F$ and $H$ an ideal of L. Suppose that $L=E \oplus \operatorname{Ann}_{L}^{1}(H)$, where $E$ is a suitable subspace such that $\operatorname{dim}_{F} E=d$ is finite. If $\operatorname{dim}_{F} H / \operatorname{Ann}_{H}^{\mathrm{r}}(E)=t$ is finite, then $\operatorname{dim}_{F}[L, H] \leqslant d t$. 
Proof Let $\left\{e_{1}, \ldots, e_{d}\right\}$ be a basis of $E$. If $x$ is an arbitrary element of $L$, then

$$
x=\alpha_{1} e_{1}+\cdots+\alpha_{d} e_{d}+c
$$

for suitable $\alpha_{1}, \ldots, \alpha_{d} \in F$ and $c \in \operatorname{Ann}_{L}^{1}(H)$. If $h \in H$, then

$$
[x, h]=\left[\alpha_{1} e_{1}+\cdots+\alpha_{d} e_{d}+c, h\right]=\alpha_{1}\left[e_{1}, h\right]+\cdots+\alpha_{d}\left[e_{d}, h\right] .
$$

It follows that $[L, H] \leqslant\left[e_{1}, H\right]+\cdots+\left[e_{d}, H\right]$. Pick $a \in L$ and define the mapping $\iota_{a}: H \rightarrow H$ by the rule $\iota_{a}(h)=[a, h], h \in H$. By Proposition 3.2, this mapping is linear, $\operatorname{Im}\left(\iota_{a}\right)=[a, H]$ and $\operatorname{Ker}\left(\iota_{a}\right)=\operatorname{Ann}_{H}^{\mathrm{r}}(a)$. Hence

$$
[a, H]=\operatorname{Im}\left(\iota_{a}\right) \cong_{F} H / \operatorname{Ker}\left(\iota_{a}\right)=H / \operatorname{Ann}_{H}^{\mathrm{r}}(a) .
$$

Let $1 \leqslant j \leqslant d$. Since $\operatorname{Ann}_{H}^{\mathrm{r}}(E) \leqslant \operatorname{Ann}_{H}^{\mathrm{r}}\left(e_{j}\right)$,

$$
\operatorname{dim}_{F} H / \operatorname{Ann}_{H}^{\mathrm{r}}\left(e_{j}\right) \leqslant \operatorname{dim}_{F} H / \operatorname{Ann}_{H}^{\mathrm{r}}(E)=t
$$

Thus $\operatorname{dim}_{F}\left[e_{j}, H\right] \leqslant t$. This holds for every $j \operatorname{sodim} F\left(\left[e_{1}, H\right]+\cdots+\left[e_{d}, H\right]\right) \leqslant t d$ and therefore $\operatorname{dim}_{F}[L, H] \leqslant t d$, as required.

Similarly we can prove the next result.

Lemma 4.3 Let $L$ be a Leibniz algebra over a field $F$ and $H$ an ideal of L. Suppose that $L=E \oplus \operatorname{Ann}_{L}^{\mathrm{r}}(H)$, where $E$ is a suitable subspace such that $\operatorname{dim}_{F} E=d$ is finite. If $\operatorname{dim}_{F} H / \operatorname{Ann}_{H}^{1}(E)=t$ is finite, then $[H, L]$ has finite dimension and $\operatorname{dim}_{F}[H, L] \leqslant d t$.

Lemmas 4.2 and 4.3 immediately imply the following conclusion.

Corollary 4.4 Let $L$ be a Leibniz algebra over a field $F$ and $H$ an ideal of L. Suppose that $L=E \oplus \operatorname{Ann}_{L}(H)$, where $E$ is a suitable subspace such that $\operatorname{dim}_{F} E=d$ is finite. If $\operatorname{dim}_{F} H / \operatorname{Ann}_{H}(E)=t$ is finite, then $\operatorname{dim}_{F}[L, H], \operatorname{dim}_{F}[H, L] \leqslant d t$.

Corollary 4.5 Let $L$ be a Leibniz algebra over a field $F$ and $H$ an ideal of L. Suppose that $\operatorname{codim}_{F} \operatorname{Ann}_{L}(H)=d$ is finite. If $\operatorname{dim}_{F} H /(H \cap \zeta(L))=t$ is finite, then $\operatorname{dim}_{F}[L, H], \operatorname{dim}_{F}[H, L] \leqslant d t$.

Indeed, $\operatorname{Ann}_{H}(E) \geqslant H \cap \zeta(L)$, so that $\operatorname{dim}_{F} H / \operatorname{Ann}_{H}(E) \leqslant t$.

Proof of Theorem A Let

$$
\{0\}=Z_{0} \leqslant Z_{1} \leqslant \cdots \leqslant Z_{k-1} \leqslant Z_{k}=Z
$$

be the upper central series of $L$. We proceed by induction on $k$. If $k=1$, then the first center $Z_{1}$ has finite codimension $d$ and it suffices to apply Corollary B1 to obtain that $\gamma_{2}(L)=[L, L]$ has dimension at most $d^{2}$. 
Assume that $k>1$ and we have already proved that

$$
\operatorname{dim}_{F} \gamma_{k}\left(L / Z_{1}\right) \leqslant 2^{k-2} d^{k}=t
$$

By Corollary 2.5, $\gamma_{k}\left(L / Z_{1}\right)=\left(\gamma_{k}(L)+Z_{1}\right) / Z_{1}$. Put $K / Z_{1}=\gamma_{k}\left(L / Z_{1}\right)$ and $T=$ $\gamma_{k}(L)$ so that $T \leqslant K$ by Corollary 2.5. Further,

$$
\operatorname{dim}_{F} T /\left(T \cap Z_{1}\right) \leqslant \operatorname{dim}_{F} K / Z_{1} \leqslant t .
$$

By Lemma 4.1, $Z_{k} \leqslant \operatorname{Ann}_{L}(T)$ so that $\operatorname{codim}_{F} \operatorname{Ann}_{L}(T) \leqslant d$. By Corollary 4.5, $\operatorname{dim}_{F}[L, T], \operatorname{dim}_{F}[T, L] \leqslant d t$.

By Proposition 2.2, $D=[L, T]+[T, L]$ is an ideal of $L$. The factor $K / D$ is abelian hence $(T+D) / D$ is abelian. We have $\operatorname{dim}_{F} D \leqslant 2 d t$. By construction of $D$, we have $[L / D, T / D] \leqslant T / D$ and $[T / D, L / D] \leqslant T / D$. These inclusions show that $T / D \leqslant \zeta(L / D)$. Since $L / T$ is nilpotent of nilpotency class $k-1$, the latter inclusion shows that $L / D$ is nilpotent of nilpotency class at most $k$. By Corollary 2.5, $\gamma_{k+1}(L) \leqslant D$, which implies

$$
\operatorname{dim}_{F} \gamma_{k+1}(L) \leqslant 2 d t=2 d 2^{k-2} d^{k}=2^{k-1} d^{k+1},
$$

as required.

Using similar arguments we can obtain Corollary A1 for Lie algebras.

\section{References}

1. Albeverio, S., Ayupov, S.A., Omirov, B.A.: On nilpotent and simple Leibniz algebras. Commun. Algebr. 33(1), 159-172 (2005)

2. Albeverio, S., Omirov, B.A., Rakhimov, I.S.: Varieties of nilpotent complex Leibniz algebras of dimension less than five. Commun. Algebr. 33(5), 1575-1585 (2005)

3. Ayupov, Sh.A., Omirov, B.A.: On Leibniz algebras. In: Khakimdjanov, Yu., Goze, M., Ayupov, Sh.A. (eds.) Algebra and Operators Theory, pp. 1-12. Kluwer, Dordrecht (1998)

4. Ayupov, Sh.A., Omirov, B.A.: On some classes of nilpotent Leibniz algebras. Sib. Math. J. 42(1), 15-24 (2001)

5. Baer, R.: Endlichkeitskriterien für Kommutatorgruppen. Math. Ann. 124, 161-177 (1952)

6. Barnes, D.W.: Schunck classes of soluble Leibniz algebras. Commun. Algebr. 41(11), 4046-4065 (2013)

7. Batten Ray, C., Combs, A., Gin, N., Hedges, A., Hird, J.T., Zack, L.: Nilpotent Lie and Leibniz algebras. Commun. Algebr. 42(6), 2404-2410 (2014)

8. Blokh, A.: A generalization of the concept of a Lie algebra. Soviet Math. Dokl. 6, 1450-1452 (1965)

9. Blokh, A.: Cartan-Eilenberg homology theory for a generalized class of Lie algebras. Soviet Math. Dokl. 8, 824-826 (1967)

10. Blokh, A.: A certain generalization of the concept of Lie algebra. Algebra and Number Theory, Moskov. Gos. Ped. Inst. Učen. Zap 375, 9-20 (1971) (in Russian)

11. Butterfield, J., Pagonis, C. (eds.): From Physics to Philosophy. Cambridge University Press, Cambridge (1999)

12. Casas, J.M., Pirashvili, T.: Ten-term exact sequence of Leibniz homology. J. Algebr. 231(1), 258-264 (2000)

13. Dobrev, V. (ed.): Lie Theory and Its Applications in Physics. Springer Proceedings in Mathematics \& Statistics, vol. 36. Springer, Tokyo (2013) 
14. Duplij, S., Wess, J. (eds.): Noncommutative Structures in Mathematics and Physics. NATO Science Series II: Mathematics, Physics and Chemistry, vol. 22. Kluwer, Dordrecht (2001)

15. Fialowski, A., Khudoyberdiyev, A.Kh., Omirov, B.A.: A characterization of nilpotent Leibniz algebras. Algebr. Represent. Theory 16(5), 1489-1505 (2013)

16. Frabetti, A.: Leibniz homology of dialgebras of matrices. J. Pure Appl. Algebra 129(2), 123-141 (1998)

17. Loday, J.-L.: Une version non commutative des algèbres de Lie: les algèbres de Leibniz. Enseign. Math. 39(3-4), 269-293 (1993)

18. Loday, J.-L., Pirashvili, T.: Universal enveloping algebras of Leibniz algebras and (co)homology. Math. Ann. 296(1), 139-158 (1993)

19. Neumann, B.H.: Groups with finite classes of conjugate elements. Proc. London Math. Soc. s3-1, 178-187 (1951)

20. Patsourakos, A.: On nilpotent properties of Leibniz algebras. Commun. Algebr. 35(12), 3828-3834 (2007)

21. Pirashvili, T.: On Leibniz homology. Ann. Inst. Fourier (Grenoble) 44(2), 401-411 (1994)

22. Stewart, I.N.: Verbal and marginal properties of non-associative algebras. Proc. London Math. Soc. s3-28, 129-140 (1974)

23. Vaughan-Lee, M.R.: Metabelian BFC p-groups. J. London Math. Soc. s2-5(4), 673-680 (1972) 